\title{
X-ray Magnetic Circular Dichroism Sum Rule Analysis of the Blue Copper Site in Plastocyanin. A Probe of Orbital and Spin Angular Momentum
}

\author{
Hongxin Wang, ${ }^{\dagger, \star}$ C. Bryant,,,+ D. W. Randall,, L. B. LaCroix, ${ }^{\S}$ E. I. Solomon, ${ }^{\S}$ \\ M. LeGros,,$*$ and S. P. Cramer $*, \hbar$ \\ Department of Applied Science, University of California, Davis, California 95616, Physical Biosciences \\ Division, Lawrence Berkeley National Laboratory, Berkeley, California 94720, and Department of Chemistry, \\ Stanford University, Stanford, California 94305
}

Received: April 30, 1998; In Final Form: July 21, 1998

\begin{abstract}
$\mathrm{Cu}$ L edge X-ray magnetic circular dichroism (XMCD) spectroscopy has been used to study the electronic structure of the blue copper site in plastocyanin. Application of the orbital angular momentum sum rule leads to an experimental (Cu 3d specific) orbital moment $\left\langle L_{z}\right\rangle$ of $0.07 \pm 0.02 \hbar / \mathrm{Cu}$ atom. This compares to $0.054 \hbar$ derived from the EPR measurements and $0.059 \hbar / \mathrm{Cu}$ atom from a SCF-X $\alpha-S W$ calculation. Application of the spin angular momentum sum rule leads to an experimental spin moment $\left\langle S_{z}\right\rangle$ of $0.18 \pm$ $0.02 \hbar / \mathrm{Cu}$ atom, compared to $0.21 \hbar / \mathrm{Cu}$ atom by SCF-X $\alpha-\mathrm{SW}$. XMCD sum rule analysis should be a useful probe of electronic structure for many other problems in inorganic and bioinorganic chemistry.
\end{abstract}

\section{Introduction}

X-ray magnetic circular dichroism (XMCD) has recently emerged as a powerful element and site-specific probe of electronic and magnetic structure. ${ }^{1,2}$ The development of ligand field multiplet calculations ${ }^{3}$ and of X-ray "sum rules" ${ }^{4-6}$ allows detailed predictions to be made about orbital and spin angular momentum for particular elements and oxidation states in complex samples. Although XMCD applications are now common in materials science and especially magnetic technology, ${ }^{7}$ its use in biological and inorganic chemistry ${ }^{8}$ has been hindered by technical problems associated with the spectroscopy of dilute and paramagnetic metal centers. In this paper we present XMCD spectra for the blue $\mathrm{Cu}$ site in plastocyanin, $, 9,10$ recorded with a new dilution refrigerator system and 30-element fluorescence detector. ${ }^{11,12}$ Plastocyanin is an ideal system for testing fluorescence-detected XMCD sum rule analysis because its electronic structure is well understood from a variety of experimental and theoretical approaches. ${ }^{9}{ }^{10}$ The half-occupied

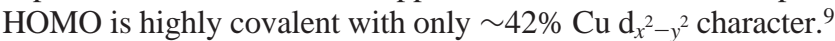
Our XMCD results are compared with EPR data and electronic structure calculations, and a variety of potential applications are discussed.

\section{Experimental Section}

The sample, plastocyanin, was isolated from spinach by previously published methods ${ }^{13}$ to a final concentration of 0.8 $\mathrm{mM}$ in $\mathrm{pH} 7$ potassium phosphate buffer. A drop $(20-50 \mu \mathrm{L})$ of the sample was placed on a gold-plated copper sample holder and allowed to dry over a period of $2 \mathrm{~h}$ at room temperature in air. During the experiment, the time of beam-on was controlled carefully to minimize the photoreduction of $\mathrm{Cu}(\mathrm{II})$ to $\mathrm{Cu}(\mathrm{I})$. In the last spectra taken, we estimate less than $10 \% \mathrm{Cu}(\mathrm{I})$ presence in plastocyanin by monitoring the changes in the minor L edge

\footnotetext{
* To whom correspondence should be addressed.

University of California.

$\doteqdot$ Lawrence Berkeley National Laboratory.

$\S$ Stanford University.
}

feature (at about $938 \mathrm{eV}$ ). On the other hand, $\mathrm{Cu}(\mathrm{I})$ has no XMCD effect.

The XMCD experiments were performed at the SSRL bend magnet beamline 8-2 using the 1100 line/mm grating. Elliptically polarized X-rays were obtained by moving the first mirror above or below the electron orbit. ${ }^{14}$ On the basis of previous calibration experiments on polarized ferromagnetic samples, the optimum XMCD signal was obtained at a beam takeoff angle corresponding to a circular polarization of $85 \pm 5 \%$. The energy resolution is estimated to be $1 \mathrm{eV}$ at the $\mathrm{Cu} \mathrm{L}$ edge with these elliptically polarized X-ray beams (with both slits at $50 \mu \mathrm{m}$ ). The XMCD apparatus uses a $76 \mathrm{~cm}$ split-coil $2 \mathrm{~T}$ superconducting magnet surrounded by a UHV chamber maintained at $\sim 5 \times 10^{-9}$ Torr. A 30-element windowless Ge fluorescence detector ${ }^{11,15}$ is inserted horizontally between the two coils, perpendicular to the photon beam path. The coldfinger of a $\mathrm{He}^{3} / \mathrm{He}^{4}$ dilution refrigerator enters the magnet bore from the top of the chamber, and the sample is attached to the coldfinger at the center of the magnet bore. Samples were introduced into the magnet bore through a vacuum load lock and screwed into the coldfinger using a removable sample insertion device. The lowest operating temperature observed in our experiments was on the order of $400 \mathrm{mK}$, as measured by a carbon resistance thermometer and by a magnetization curve.

Individual scans were taken over the $\mathrm{Cu} \mathrm{L}$ edges using 0.2 $\mathrm{eV}$ steps at $6 \mathrm{~s}$ per point integration time. One set of 20 scans was taken with right circular polarization. Every two scans, the magnetic field was switched between -2 and $+2 \mathrm{~T}$. A second set of 20 scans was then taken with left circular polarization, again alternating the sign of the magnetic field. The apparent XMCD effect did reverse with opposite beam polarization. For measurement of the magnetization curves, a similar procedure was followed, collecting only the $\mathrm{L}_{3}$ edge region, using between 0.1 and $2.0 \mathrm{~T}$. The raw data (fluorescence signal, $F$ ) were divided by an internal $I_{0}$ monitored by the intensity of oxygen $\mathrm{K}$ fluorescence (proportional to the beam flux). 


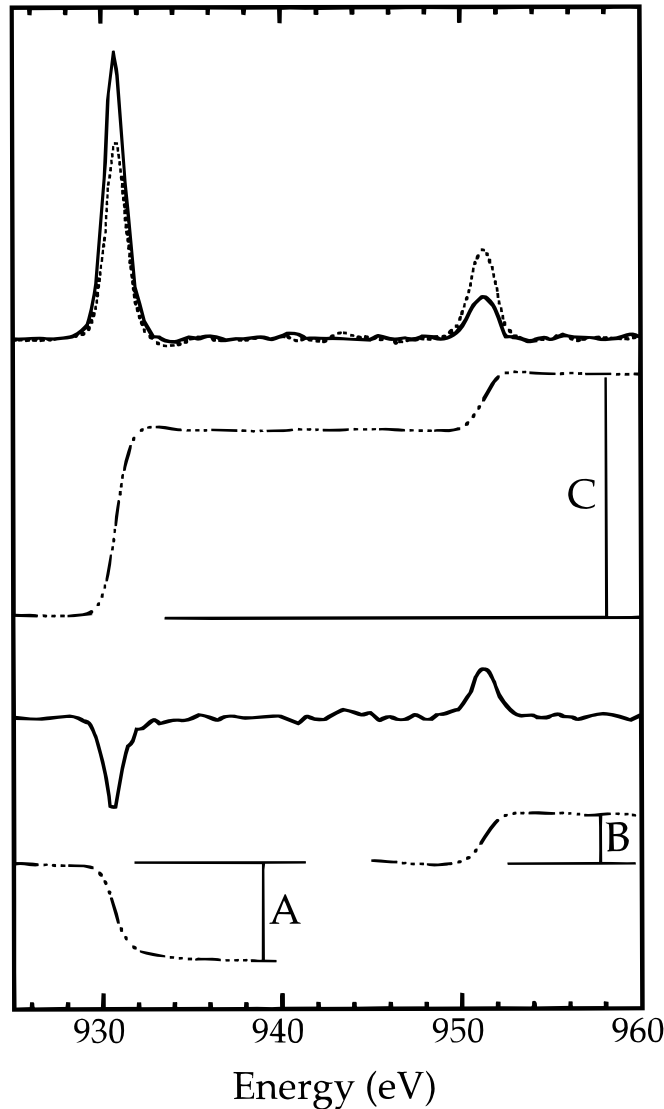

Figure 1. (Upper portion) X-ray polarization-corrected, backgroundsubtracted $\mathrm{L}_{2,3}$ edge absorption spectra of $\mathrm{Cu}(\mathrm{II})$ in plastocyanin, with incident photon spin parallel $\left(I^{\Uparrow \uparrow}\right.$, solid line) and antiparallel $\left(I^{\Uparrow \downarrow}\right.$, dotted line) to the spin of $\mathrm{Cu}$; (lower portion) XMCD spectrum $\left(I^{\Uparrow}-I^{\uparrow}\right)$ for $\mathrm{Cu}$ (II) in plastocyanin (solid line). The dot-dash line underneath the absorption spectra is the integral curve $(\mathrm{C})$ of the average absorption $\left(I^{\Uparrow}+I^{\Uparrow}\right) / 2$ over the whole L edge while the lines underneath the XMCD spectrum are the integrals $(A$ and $B)$ of the XMCD spectrum over $\mathrm{L}_{3}$ and $\mathrm{L}_{2}$, respectively.

The final spectrum is the sum of 20 individual $\left(F / I_{0}\right)$ sets for each side, with the X-ray photon angular momentum either parallel $\left(I^{\Uparrow \uparrow}\right)$ or antiparallel, $\left(I^{\Uparrow \downarrow}\right)$ to that of the C(II) majority $3 \mathrm{~d}$ spin. Each of the averaged spectra were first normalized to the edge jump between the pre- $\mathrm{L}_{3}$ and post- $\mathrm{L}_{2}$ edge and then corrected for the small energy offset between spectra recorded on opposite polarization. A cubic polynomial was then fit to the raw data $\left(F / I_{0}\right)$ in the pre- $\mathrm{L}_{3}$ and post- $\mathrm{L}_{2}$ regions to simulate the nonresonant (background) X-ray absorption. They were then subtracted from the overall spectrum leaving only the resonant component of the absorption signal. Finally the spectra were corrected for the fact that the illuminating X-rays were only $85 \%$ polarized.

\section{Results and Discussion}

The polarization-corrected $\mathrm{L}$ edge excitation spectra ${ }^{14,15}$ for $\mathrm{Cu}$ in plastocyanin, taken with the X-ray photon angular momentum parallel $\left(I^{\Uparrow}\right)$ and antiparallel $\left(I^{\uparrow}\right)$ to the sample magnetization, are reported in Figure 1. Also shown is the difference in the polarization-corrected fluorescence signals $\left(I^{\uparrow}-I^{\uparrow}\right)-$ the XMCD spectrum. At the $\mathrm{L}_{3}$ edge peak there is a $42 \%$ decrease in fluorescence intensity between opposite polarizations, while at the $\mathrm{L}_{2}$ edge the effect is of the opposite sign. Although a $100 \%$ effect is predicted ${ }^{16}$ at the $\mathrm{L}_{2}$ edge for $D_{4 h}$ molecules aligned with their 4-fold axes along the magnetic field, for randomly oriented molecules a $50 \%$ effect is pre-

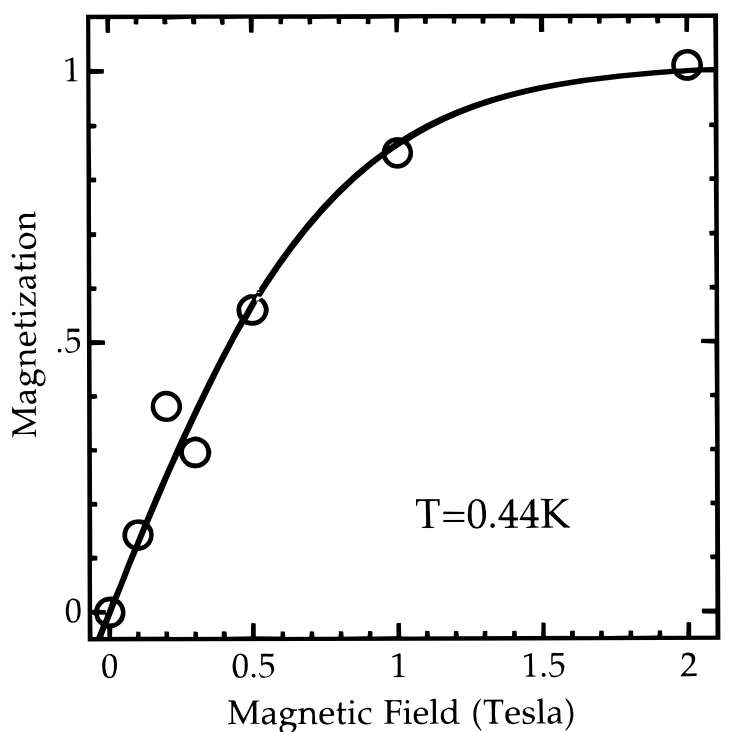

Figure 2. XMCD magnetization curve for $\mathrm{Cu}$ (II) in plastocyanin. The open circles represent the experimental data and the solid line is a Brillouin function fit to the data, leading to a temperature of $0.44 \mathrm{~K}$ and a magnetic saturation of $99.5 \%$ at $2 \mathrm{~T}$.

dicted, ${ }^{16}$ similar to the current finding. Since application of the sum rules requires knowledge of the degree of sample magnetization, we also measured the field dependence of the XMCD effect (Figure 2). Least-squares fitting with a Brillouin function for a $J=1 / 2$ system gives a sample temperature of $0.44 \mathrm{~K}$, from which we deduce $99.5 \%$ magnetic saturation at 2 $\mathrm{T}$.

When applied to $3 \mathrm{~d}$ transition metal L edges, the orbital and spin sum rules ${ }^{4-6}$ relate integrated XMCD intensities to element specific projections of the $3 \mathrm{~d}$ orbital angular momentum $\left\langle L_{z}\right\rangle$, the $3 \mathrm{~d}$ spin angular momentum $\left\langle S_{z}\right\rangle$, and a magnetic dipole term $\left\langle T_{z}\right\rangle$, as summarized in eqs 1 and 2, respectively. Stöhr and König ${ }^{7}$ have shown that the $\left\langle T_{z}\right\rangle$ term angular averages to zero in "powder" samples, so this term has been omitted from our equations.

$$
\begin{gathered}
\left\langle L_{z}\right\rangle=-2 n_{\mathrm{h}} \cdot \frac{\int_{\mathrm{L}_{3}+\mathrm{L}_{2}}\left[I^{\uparrow}-I^{\uparrow \downarrow}\right] \mathrm{d} \omega}{\int_{\mathrm{L}_{3}+\mathrm{L}_{2}}\left[I^{\uparrow}+I^{0}+I^{\uparrow}\right] \mathrm{d} \omega} \\
\left\langle S_{z}\right\rangle=-\frac{3}{2} n_{\mathrm{h}} \cdot \frac{\int_{\mathrm{L}_{3}}\left[I^{\Uparrow \uparrow}-I^{\uparrow \downarrow}\right] \mathrm{d} \omega-2 \int_{\mathrm{L}_{2}}\left[I^{\uparrow}-I^{\uparrow}\right] \mathrm{d} \omega}{\int_{\mathrm{L}_{3}+\mathrm{L}_{2}}\left[I^{\uparrow}+I^{0}+I^{\uparrow \downarrow}\right] \mathrm{d} \omega}
\end{gathered}
$$

Here $n_{\mathrm{h}}$ represents the number of $3 \mathrm{~d}$ vacancies in the metal ion, $\omega$ is the X-ray frequency, and $I^{0}$ refers to the absorption for $\mathrm{X}$-rays linearly polarized along the magnetization direction; as is customary, we approximate this by $\left(I^{\Uparrow}+I^{\uparrow}\right) / 2$. It is common in the XMCD literature ${ }^{1,7,15}$ to refer to the first integral in the numerator of eq 2 as " $A$ ", the second integral as " $B$ ", and the denominator as " $3 C$ ". The necessary integrals are illustrated in Figure 1. One can thus re-express the sum rules in the following manner, in units of $\hbar$ /atom:

$$
\left\langle L_{z}\right\rangle=-n_{\mathrm{h}} \frac{2(A+B)}{3 C} \quad\left\langle S_{z}\right\rangle=-n_{\mathrm{h}} \frac{A-2 B}{2 C}
$$

After normalizing the overall $\mathrm{Cu} \mathrm{L}$ edge jump between 920 and $1050 \mathrm{eV}$ to unity, ${ }^{17}$ average numerical values for $A$ $(-3.08 \pm 0.3), B(1.35 \pm 0.2)$, and $C(7.27 \pm 0.3)$ were 
obtained, where the errors refer to the degree of experimental reproducibility. The integrated total absorption intensity $(C)$ is slightly higher than in previous work, and it leads to a value of 0.44 for $n_{\mathrm{h}}$, as compared to the previous value of $0.41 .^{17}$ This value is still in good agreement with theoretical calculations that predict a highly covalent site. ${ }^{9,10}$ From eq $1,\left\langle L_{z}\right\rangle$ is found to be $0.07 \pm 0.02 \hbar / \mathrm{Cu}$ atom, while from eq 2 we derive 0.18 $\pm 0.02 \hbar / \mathrm{Cu}$ atom for $\left\langle S_{z}\right\rangle$.

To complement these experimental predictions for $\left\langle S_{z}\right\rangle$ and $\left\langle L_{z}\right\rangle$ values, a series of SCF-X $\alpha-\mathrm{SW}$ calculations ${ }^{18,19}$ was performed. The wave functions were orthonormalized using the Gram-Schmidt technique to generate a complete basis set. The spin-orbit interaction matrix is given by $H_{i j}=E_{i j} \delta_{i j}+$ $\sum_{a}\left\langle i_{a}\left|\lambda_{a} L \cdot S\right| j_{a}\right\rangle$, where the sum over $a$ accounts for spin-orbit coupling for both $\mathrm{Cu}$ and $\mathrm{S}, i$ and $j$ are two basis functions, and $\lambda$ is the spin-orbit coupling constant $\left(-828 \mathrm{~cm}^{-1}\right.$ for $\mathrm{Cu}$ and $-382 \mathrm{~cm}^{-1}$ for S). Diagonalizing this matrix gives the spinorbit corrected wave functions. Calculations of $\left\langle L_{z}\right\rangle$ used linear combinations of the spin-orbit corrected ground-state wave functions in which $S_{q}$, where $q=x, y$, or $z$, was diagonal. ${ }^{19}$

We first evaluated $\left\langle L_{z}\right\rangle$ for a hypothetical system where all orbitals are treated as having pure $d$ character and are at the energies observed for plastocyanin. ${ }^{18,19}$ This yielded an orientation averaged $\left\langle L_{z}\right\rangle$ of $0.139 / \mathrm{Cu}$ atom, which becomes $0.058 / \mathrm{Cu}$ atom when scaled according to the covalency of plastocyanin. Also, on the basis of the same methodology, but starting with covalent wave functions from a converged SCF-X $\alpha-S W$ calculation, ${ }^{18}$ we directly calculate a $\mathrm{Cu} 3 \mathrm{~d}$ specific orientationaveraged $\left\langle L_{z}\right\rangle$ value of $0.059 / \mathrm{Cu}$ atom. Our XMCD result is thus in good agreement with the calculated values of $\left\langle L_{z}\right\rangle$, which include covalency in the wave functions.

The XMCD sum rule value of $\left\langle L_{z}\right\rangle$ can also be compared to an angle-averaged $\left\langle L_{z}\right\rangle$ determined from the deviation of the EPR $g$-values for plastocyanin $\left(g_{x}=2.047, g_{y}=2.059, g_{z}=\right.$ 2.226 $)^{20}$ from $g_{\mathrm{e}}=2.0023$ using $L_{q}=\left(g_{q}-g_{\mathrm{e}}\right) / 2$. The EPR derived value is 0.054 , which reflects orbital angular momentum contributions from the entire spin system. This value is slightly less than the $\mathrm{Cu}$ specific experimental and calculated values, and it is consistent with the possible $\mathrm{S}$ ligand influence to the covalent spin system. The sum rule values for $\left\langle S_{z}\right\rangle(0.18 \pm$ $0.02 \hbar / \mathrm{Cu}$ atom) and $\left\langle L_{z}\right\rangle /\left\langle S_{z}\right\rangle(0.39)$ are also in reasonable agreement with the SCF-X $\alpha-\mathrm{SW}$ calculation $\left(\left\langle S_{z}\right\rangle=0.21 \hbar / \mathrm{Cu}\right.$ atom and $\left\langle L_{z}\right\rangle\left\langle\left\langle S_{z}\right\rangle=0.31\right)$. In principle, the discrepancy in $\left\langle S_{z}\right\rangle$ could come from a number of sources, including the $3 \mathrm{~d}$ hole measurement, fluorescence yield (FY) variation over L edge, ${ }^{21,22}$ and partial orientation of the dehydrated sample. Better XMCD spectra on a range of systems are needed to see if the accuracy of the method can be further improved.

\section{Summary}

The XMCD values for $\left\langle L_{z}\right\rangle$ and $\left\langle S_{z}\right\rangle$ for $\mathrm{Cu}(\mathrm{II})$ in plastocyanin are within $15 \%$ of those from SCF-X $\alpha-S W$ calculation. Although there is some discrepancy with the results from EPR measurements, XMCD and EPR measure different average properties. These XMCD results demonstrate that sum rule analysis could be a useful method to study electronic structure in paramagnetic inorganic systems. Using X-ray spectroscopy to characterize local charge and momentum distributions should be a valuable complement to other structure determination methods in biological sciences.

Acknowledgment. This research was supported by the National Institutes of Health (GM-44380, GM-18812), the National Science Foundation (DMB-9107312, BIR-9317942, and CHE-9528250), and the Department of Energy, Office of Biological and Environmental Research. The Stanford Synchrotron Radiation Laboratory is supported by the Department of Energy, Office of Basic Energy Sciences. We also thank Dr. Mahesh G. Samant of IBM, San Jose, for his assistance in the use of beamline $8-2$.

\section{References and Notes}

(1) Stöhr, J.; König, H. Phys. Rev. Lett. 1995, 75, 3748.

(2) Weller, D.; Wu, Y.; Stöhr, J.; Samant, M. G.; Hermsmeier, B. D.; Chappert, C. Phys. Rev. B 1994, 49, 12888.

(3) de Groot, F. M. F. J. Electron Spectrosc. Relat. Phenom. 1994 67,529

(4) Thole, B. T.; Carra, P.; Sette, F.; van der Laan, G. Phys. Rev. Lett. 1992, 68, 1943

(5) Carra, P.; Thole, B. T.; Altarelli, M.; Wang, X. D. Phys. Rev. Lett. 1993, 70, 694

(6) Altarelli, M. Phys. Rev. B 1993, 47, 597.

(7) Stöhr, J. J. Electron Spectrosc. Relat. Phenom. 1995, 75, 253.

(8) van Elp, J.; George, S. J.; Chen, J.; Peng, G.; Chen, C. T.; Tjeng, L. H.; Meigs, G.; Lin, H. J.; Zhou, Z. H.; Adams, M. W. W.; Searle, B. G.; Cramer, S. P. Proc. Natl. Acad. Sci. U.S.A. 1993, 90, 9664.

(9) Solomon, E. I.; Penfield, K. W.; Gewirth, A. A.; Lowery, M. D.; Shadle, S. E.; Guckert, J. A.; Lacroix, L. B. Inorg. Chim. Acta 1996, 243, 67.

(10) Holm, R. H.; Kennephol, P.; Solomon, E. I. Chem. Rev. 1996, 96 2239

(11) Bryant, C.; Legros, M.; Wang, H.; Chen, J.; Jirmanus, M. Z.; Cramer, S. P., manuscript in preparation.

(12) Ralston, C.; Yocum, M.; Tench, O.; Colaresi, J.; Cramer, S. P., manuscript in preparation.

(13) Ellefson, W. Li; Ulrich, E. A.; Krogmann, D. W. In Methods in Enzymology, Dietro, S., Ed.; McGraw-Hill: New York, 1980; Vol. 69, p 223.

(14) Nakajima, R. Ph.D. Thesis, Stanford University, 1997.

(15) Cramer, S. P.; Chen, J.; George, S. J.; van Elp, J.; Moore, J.; Tench, O.; Colaresi, J.; Yocum, M.; Mullins, O. C.; Chen, C. T. Nucl. Instrum. Methods A 1992, 319, 285.

(16) van Elp, J.; Searle, B. G. J. Electron Spectrosc. Relat. Phenom. 1997, 86, 93.

(17) George, S. J.; Lowery, M. D.; Solomon, E. I.; Cramer, S. P. J. Am. Chem. Soc. 1993, 115, 2968.

(18) LaCroix, L. B.; Shadle, S. E.; Wang, Y.; Averill, B. A.; Hedman, B.; Hodgson, K. O.; Solomon, E. I. J. Am. Chem. Soc. 1996, 118, 7755.

(19) Penfield, K. W.; Gewirth, A. A.; Solomon, E. I. J. Am. Chem. Soc. 1985, 107, 4519.

(20) Penfield, K. W.; Gay, R. R.; Himmelwright, R. S.; Eickman, N. C.; Norris, V. A.; Freeman, H. C.; Solomon, E. I. J. Am. Chem. Soc. 1981 103,4382

(21) de Groot, F. M. F.; Arrio, M. A.; Sanictavit, Ph.; Cartier, Ch.; Chen, C. T. Solid State Commun. 1994, 92, 991.

(22) Wang, H.; Peng, G.; Miller, L. M.; Scheuring, E. M.; George, S J.; Chance, M. R.; Cramer, S. P. J. Am. Chem. Soc. 1997, 119, 4921.

(23) Tirsell, K. G.; Karpenko, V. P. Nucl. Instrum. Methods 1990, A291, 